\title{
From recommendation to action: psychosocial factors influencing physician intention to use Health Technology Assessment (HTA) recommendations
}

\author{
Marie-Pierre Gagnon*1, Emília Sánchez² and Joan MV Pons²
}

Address: ${ }^{1}$ Evaluative Research Unit, Quebec University Hospital Centre, Quebec, Canada and ${ }^{2}$ Catalan Agency for Health Technology Assessment and Research (CAHTAR), Barcelona, Spain

Email: Marie-Pierre Gagnon* - marie-pierre.gagnon@mfa.ulaval.ca; Emília Sánchez - esanchez@aatrm.catsalut.net;

Joan MV Pons - jpons@aatrm.catsalut.net

* Corresponding author

Published: 3I March 2006

Implementation Science2006, 1:8 doi:10.1 186/1748-5908-I-8
Received: 16 December 2005

Accepted: 31 March 2006

This article is available from: http://www.implementationscience.com/content/l/I/8

(c) 2006Gagnon et al; licensee BioMed Central Ltd.

This is an Open Access article distributed under the terms of the Creative Commons Attribution License (http://creativecommons.org/licenses/by/2.0), which permits unrestricted use, distribution, and reproduction in any medium, provided the original work is properly cited.

\begin{abstract}
Background: Evaluating the impact of recommendations based upon health technology assessment (HTA) represents a challenge for both HTA agencies and healthcare policy-makers. Using a psychosocial theoretical framework, this study aimed at exploring the factors affecting physician intention to adopt HTA recommendations. The selected recommendations were prioritisation systems for patients on waiting lists for two surgical procedures: hip and knee replacement and cataract surgery.

Methods: Determinants of physician intention to use HTA recommendations for patient prioritisation were assessed by a questionnaire based upon the Theory of Interpersonal Behaviour. A total of 96 physicians from two medical specialties (ophthalmology and orthopaedic surgery) responded to the questionnaire (response rate 44.2\%). A multiple analysis of variance (MANOVA) was performed to assess differences between medical specialties on the set of theoretical variables. Given the main effect difference between specialties, two regression models were tested separately to assess the psychosocial determinants of physician intention to use HTA recommendations for the prioritisation of patients on waiting lists for surgical procedures.
\end{abstract}

Results: Factors influencing physician intention to use HTA recommendations differ between groups of specialists. Intention to use the prioritisation system for patients on waiting lists for cataract surgery among ophthalmologists was related to attitude towards the behaviour, social norms, as well as personal normative beliefs. Intention to use HTA recommendations for patient prioritisation for hip and knee replacement among orthopaedic surgeons was explained by: perception of conditions that facilitated the realisation of the behaviour, personal normative beliefs, and habit of using HTA recommendations in clinical work.

Conclusion: This study offers a model to assess factors influencing the intention to adopt recommendations from health technology assessment into professional practice. Results identify determinant factors that should be considered in the elaboration of strategies to support the implementation of evidence-based practice, with respect to emerging health technologies and modalities of practice. However, it is important to emphasise that behavioural determinants of evidence-based practice vary according to the specific technology considered. Evidence-based implementation of HTA recommendations, as well as other evidence-based practices, should build on a theoretical understanding of the complex forces that shape the practice of healthcare professionals. 


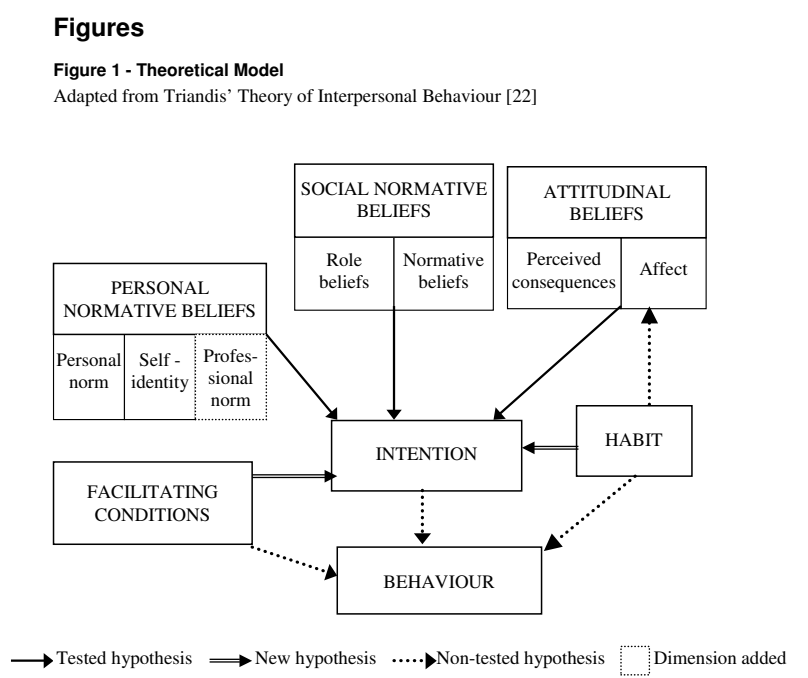

Figure I

Theoretical Model Adapted from Triandis' Theory of Interpersonal Behaviour [22]

\section{Background}

Health Technology Assessment (HTA) is a multidisciplinary field of applied research that aims to provide the best evidence available on health technologies in order to inform policy-making $[1,2]$. In HTA, the definition of health technology is broad and encompasses all methods used by health professionals to promote health, prevent and treat disease, and improve rehabilitation and longterm care [3].

It is generally recognised that there is a gap between the production of scientific evidence and its utilisation to inform decision-making, [4], and this also applies to the field of HTA [5-8]. Despite growing interest in HTA, both in the governmental and scientific spheres, few efforts have been made to assess HTA impact on decision-making at different levels of the healthcare system [6]. Furthermore, there is a paucity of specific methodologies and tools to assess the uptake of HTA recommendations [5].

At the health policy level, previous work has reported that HTA recommendations could influence decision-making [9-11]. According to a multi-method study of the implementation of guidance issued by the National Institute for Clinical Excellence (NICE) in England and Wales, [12] the extent to which HTA led to changes in practices was variable. Moreover, a review of HTA utilisation in four European countries indicates that, in spite of substantial human and financial investments, the actual impact of HTA on policy-making was still limited [13].
Hivon and collaborators have explored end-users' perceptions and use of HTA recommendations [14]. Their findings indicate that knowledge produced from HTA was not always used directly in decision-making, but could serve various purposes. According to these authors, HTA recommendations could have an instrumental, conceptual or symbolic use in decision-making [14]. Instrumental use implies that recommendations from HTA are directly translated into a decision. HTA recommendations also can have a conceptual use by providing a knowledge basis for debate and positioning. Finally, decision-makers can make a symbolic use of HTA recommendations, using them to reinforce or justify their decisions. Thus, studies assessing HTA utilisation should explore the various purposes that scientific evidence can serve in the formulation of healthcare policies.

At the healthcare organisations level, the implementation of hospital-based HTA activities could represent a strategy to improve practices [7]. Hospital-based HTA is believed to provide scientific evidence that is context-relevant, which would eventually lead to the adoption of best practices $[15,16]$. Experiences with HTA activities in hospitals have reported positive impact on resources and costs [15]. Other experiences of decentralized HTA activities include the implementation of units dedicated to HTA at the regional health authority level, such as in Health Regions in Canada [17]. However, evidence is still lacking on how HTA activities should be integrated within healthcare organisations [18].

Until now, little is known about the implementation of HTA recommendations at the individual level, i.e. in the daily practice of healthcare professionals. However, the literature on physician adoption of scientific evidence and interventions to improve it is extensive [19]. Thus, it is possible to draw from this body of knowledge in order to better understand the mechanisms involved in the adoption of HTA recommendations into clinical practices.

\section{Theoretical foundations}

In the field of social psychology, various theories and models have been proposed to understand what influences the adoption of behaviours. Triandis' Theory of Interpersonal Behaviour (TIB) [20] encompasses many of the behavioural determinants found in other psychosocial theories, such as the Theory of Planed Behaviour [21] and the Social Cognitive Theory [22]. Moreover, the TIB also considers cultural, social, and moral factors that are particularly important in the study of specific groups, such as healthcare professionals $[23,24]$.

A schema adapted from the TIB is presented in Figure 1. According to this theory, human behaviour is formed by three components: intention, facilitating conditions, and 
habit. Intention refers to the individual's motivation regarding the performance of a given behaviour. Facilitating conditions represent perceived factors in the environment that can ease or impede the realisation of a given behaviour. Habit refers to how routine a given behaviour has become, i.e. the frequency of its occurrence. Habit directly influences the behaviour, but can also have an influence on affect. However, this hypothesis was not tested in the present study.

In the TIB, the behavioural intention is formed by attitudinal as well as normative beliefs. Attitudinal beliefs comprise two dimensions: affect and perceived consequences. Affect represents an emotional state that the performance of a given behaviour evokes for an individual. It is considered as the affective perceived consequences of the behaviour, whereas perceived consequences refer to individual's perception of the instrumental consequences of the behaviour.

The TIB also distinguishes between two normative dimensions: social and personal. Social normative beliefs are formed by normative and role beliefs. Normative beliefs consist of the internalisation by an individual of referent people's or groups' opinions about the realisation of the behaviour, whereas role beliefs reflect the extent to which an individual thinks someone of his or her age, gender, and social position should or should not behave. With respect to the personal normative beliefs, personal norm represents the feeling of personal obligation regarding the performance of a given behaviour, whereas self-identity refers to the degree of congruence between the individual's perception of self and the characteristics associated with the realisation of the behaviour.

For the purpose of this study, modifications were brought to the original TIB model. These modifications were consistent with a previous study that has adapted the TIB to understand healthcare professional behaviour [24]. First, the dependent variable of interest in this study is the behavioural intention rather than the behaviour. Thus, the original relationships between facilitating conditions and behaviour, as well as between habit and behaviour have been modified to explore the influence of these constructs on the behavioural intention. These relationships are consistent with previous studies that used the TIB to predict behavioural intention [25-27].

Furthermore, in an effort to better adapt the TIB to health professional behaviour, another dimension was added to the personal normative beliefs - the professional norm. This variable is related to the integration by the self of the specific normative pressures of one's professional group. The medical profession has a particular culture and sets of norms (e.g. the Hippocratic Oath) that also influence individual physician behaviour [28]. In a previous study, adding the professional norm to the personal normative construct significantly improved the predictive validity of this construct in explaining physicians' decision to adopt a new technology [24]. This construct is also consistent with the concept of collective self, as proposed by Triandis, which corresponds to the individual's assessment of how she or he should behave given her or his belonging to a specific reference group [29]. The professional norm is considered a of the dimension of the personal normative construct since previous work has shown association between these factors [24].

To the best of our knowledge, the TIB has not previously been applied to the study of the adoption of evidencebased recommendations into medical practice. However, this model was successful in explaining a variety of professional behaviours, such as the adoption of information and communication technologies [24,25,30,31].

\section{Description of the study}

This study is part of a larger initiative aimed at applying a multi-dimensional theoretical framework to assess the impact of HTA recommendations on decision-making at different levels of the healthcare system. Thus, various methods were used in order to assess factors influencing the uptake of HTA recommendations at the healthcare organisation and clinical decision-making levels. HTA adoption at the organisational level was assessed through a qualitative approach by means of interviews and observations at 15 hospitals of Catalonia. The results of the qualitative study are presented elsewhere $[32,33]$.

In summary, the qualitative study indicates that factors related to the organisation and financing of the health system influence adoption of HTA recommendations at the hospital level. Furthermore, collaborations between hospitals and the HTA agency favour the integration of recommendations into organisational practices. At the professional level, the high degree of autonomy of medical specialists, the importance of peers and collegial control, and the definition of professional roles and responsibilities influence adoption of HTA recommendations.

The present article focuses on the impact of HTA recommendations at the individual level, which has been conceptualised as physician intention to use HTA recommendations to support clinical decision-making. This study was conducted as part of a postdoctoral fellowship (MPG), and the research protocol was approved by Catalonia and Quebec governments. The Catalan Agency for Health Technology Assessment and Research (CAHTAR) also reviewed the research protocol and provided support for the study. 


\section{Research Questions}

Based upon the TIB, this study aimed to answer the following questions:

1. Which psychosocial factors from the TIB (attitudinal beliefs, social normative beliefs, personal normative beliefs, facilitating conditions, habit) significantly explain the intention of physicians to adopt these recommendations into their practice?

2. Are the psychosocial factors influencing physicians' intention to adopt HTA recommendations significantly different between the two groups of specialists?

3. Do sociodemographic and professional factors (age, gender, work experience) influence physicians' intention to adopt HTA recommendations over and above the psychosocial constructs from the TIB?

\section{Methods}

\section{Selection of health technologies}

A consensus was reached with researchers from the CAHTAR to select which recommendations would be investigated. The criteria used in the selection were: 1) publication time sufficient for the HTA recommendation to have been largely disseminated; 2) recommendations representing administrative and clinical health technologies, since the literature reports important variations in factors affecting the adoption of these two types of innovations;[34] and 3) similar recommendations that would allow comparisons between cases for a greater internal validity. Thus, a total of three recommendations were selected. Two were related to clinical-administrative technologies, namely prioritisation systems for patients on waiting lists for two distinct surgical procedures - cataract surgery and hip and knee replacement. The third recommendation covered the prescription of external pump for continuous subcutaneous insulin infusion for patients with Type I diabetes. However, it was not possible to analyse the factors affecting the adoption of this recommendation quantitatively, given the limited number of endocrinologists (7) in the sample. Thus, only the recommendations regarding the two prioritisation systems were considered in the analysis of HTA recommendations' impact at the individual decision-making level.

Both recommendations proposed a scoring system to assess patient priority on waiting lists for the targeted surgical procedures. The prioritisation systems for cataract surgery and hip and knee replacement were similar, although specific scoring items were used. Their utilisation by physicians practicing in the Catalan network of public hospitals was made mandatory through an instruction issued by the Servei Català de la Salut (the Catalan Health Service) in November 2004.

\section{Development of the survey instrument}

The field of social psychology has a long tradition in the development of survey instruments based upon theoretical frameworks. In the present study, questionnaire development was based on several theorists' recommendations $[21,35,36]$. The TIB provided the conceptual constructs that were measured, but we adapted the content (i.e. wording of the questions) to the specific behaviour under study and the particular culture of the target group. This is known in anthropology as the emic-etic approach and has been recommended by psychosocial theorists in order to ensure the cultural sensitivity of a study $[37,38]$.

First, an open-ended questionnaire was prepared in order to assess the modally salient beliefs in the study population with respect to the behaviour under consideration. Salient beliefs are the first responses to come to a respondent's mind when asking an open-ended question. Therefore, modally salient beliefs are the most frequently reported beliefs regarding the attributes of performing a particular behaviour in the target group [39]. Thus, a purposive sample of 10 physicians within each medical specialty was sent a questionnaire comprising eight openended questions. Questions assessed the attitudinal, social normative and personal normative beliefs, as well as the perceived facilitating conditions and barriers with respect to using HTA recommendations to support decision-making.

Completed questionnaires were received from five ophthalmologists and seven orthopaedic surgeons. Responses were compiled for each specialty. A content analysis was performed to classify responses into thematic categories. Then the number of responses in each category was compiled, and those having a frequency of two or more were kept as the modally salient beliefs. These salient beliefs were used as the items to assess each theoretical construct of the TIB. A specific questionnaire was developed for each medical specialty, since two distinct recommendations were addressed. However, given the similitude between these recommendations, the two questionnaires used the same items to assess theoretical constructs, thus allowing for the combination of results and comparisons between groups.

The first page of the questionnaire presented the study and gave instructions to participants. A sentence indicated that returning the questionnaire implied informed consented to participate in the study. The questionnaire began with a vignette describing a clinical case for which the surgical procedure (cataract surgery or hip and knee replacement) was relevant. By referring to the case presented in the vignette, physicians were asked to answer a total of 30 questions measuring the theoretical constructs of the TIB. 
Table I: Internal consistency of theoretical constructs

\begin{tabular}{lcc}
\hline Construct & Number of items & Internal consistency (Cronbach's alpha) \\
\hline Intention & 3 & 0.85 \\
Attitudinal beliefs & 7 & 0.81 \\
Personal normative beliefs & 6 & 0.86 \\
Social normative beliefs & 6 & 0.82 \\
Facilitating conditions & 3 & 0.75 \\
Habit & 4 & 0.87 \\
\hline
\end{tabular}

Each theoretical item was assessed by a question measured on a five-point Likert scale. For example, to what extent do you agree with the following affirmation - "It would be easy for me to use CAHTAR's recommendations to support my decision in this case." 1) Totally disagree; 2) Slightly disagree; 3) Neither agree nor disagree; 4) Slightly agree; or 5) Totally agree. The only exception was for the items composing the attitudinal construct that were assessed by means of 5-point bi-polar adjective scales. For example, "For me, using CAHTAR's recommendations to support decision-making in this case would be..." 1) Very foolish; 2) Somewhat foolish; 3) Neither foolish nor wise; 4) Somewhat wise; or 5) Very wise. The number of items used to assess each theoretical construct and their internal consistency are provided in Table 1 . The Cronbach $\alpha$ was used to verify the internal consistency of theoretical constructs. As shown in Table 1, all constructs showed satisfactory internal consistency, with Cronbach $\alpha$ higher than $0.70[40]$.

Finally, socio-demographic information (age group, gender, years of clinical experience, and medical specialty) was collected at the end of the questionnaire. The questionnaire was pre-tested with two physicians of each specialty in order to assess face validity and duration. Subsequently, minor adjustments were done to the wording of some questions. The questionnaire took approximately 15 minutes to complete.

\section{Participants and setting}

A total of 15 hospitals were selected to most fully represent the various profiles of Catalan hospitals. Hospitals from the eight Catalan Health Regions were represented. The sample consisted of publicly and privately-funded hospitals (all provided services in the public system), as well as large teaching hospitals and smaller general hospitals. Heads of department or service for the targeted specialties (ophthalmology and orthopaedic surgery) were identified in each hospital as the local collaborators. The principal investigator contacted them by telephone to describe the study and solicit their participation. After receiving consent from all contacted persons, a package containing study questionnaires corresponding to the number of physicians who worked in the service was delivered to the local collaborator in each hospital. The total sample consisted of 217 physicians ( 80 ophthalmologists and 137 orthopaedic surgeons).

\section{Statistical analyses}

First, descriptive analyses of distribution were conducted. Correlations between theoretical variables and between theoretical and external variables were assessed and are reported in Table 2. All the theoretical constructs from the TIB had a significant positive association with the intention. Medical specialty was the only external variable having a significant correlation with theoretical variables. None of the external variables were significantly correlated with intention.

Second, a comparison between the two groups of specialists was performed on the set of theoretical variables using the multivariate analysis of variance (MANOVA). Given the significant differences between groups, two independent hierarchical regression models were tested in order to assess the determinants of physician intention to use HTA recommendations. The potential impact of external variables (socio-demographic and professional characteristics) on intention was tested following Pedhazur's recommendation, which consists of comparing the $\mathrm{R}^{2}$ of the model containing only theoretical variables with the $\mathrm{R}^{2}$ of a model also containing external variables [41]. No significant difference was found. We also assessed potential interaction effects of external variables by entering interaction terms between theoretical and external variables that were significantly correlated (e.g. attitude and experience in the orthopaedic surgeons group) in the regression equation [42], but no significant effect was found for the interaction terms. The final regression models were calculated by keeping only the significant predictors in the equation. All statistical analyses were performed using SPSS version 12.0. (SPSS Inc., Chicago, IL)

\section{Results \\ Descriptive statistics}

A total of 96 physicians returned completed questionnaires (35 ophthalmologists and 61 orthopaedic surgeons) for a global response rate of $44.2 \%$. Table 3 presents the sociodemographic and professional charac- 
Table 2: Zero-order correlations between theoretical and sociodemographic variables

\begin{tabular}{|c|c|c|c|c|c|c|c|c|c|}
\hline Variable & Attitude & Personal norms & Social norms & Facilitating cond. & Habit & Age & Gender & Specialty & Experience \\
\hline Intention & $0.715^{* * *}$ & $0.78 I^{* * *}$ & $0.716^{* * *}$ & $0.510 * * *$ & $0.677^{* * *}$ & 0.109 & -0.003 & -0.049 & 0.147 \\
\hline Attitude & & $0.664^{* * * *}$ & $0.754^{* * * *}$ & $0.500 * * *$ & $0.591 * * * *$ & -0.046 & 0.035 & $-0.303 * *$ & -0.038 \\
\hline Personal norms & & & $0.72 I^{* * * *}$ & $0.424 * * *$ & $0.695 * * *$ & 0.044 & 0.093 & $-0.24 I^{*}$ & 0.063 \\
\hline Social norms & & & & $0.434 * * *$ & $0.666 * * *$ & -0.033 & 0.085 & $-0.253^{*}$ & -0.007 \\
\hline Facilitating cond. & & & & & $0.422^{* * * *}$ & -0.098 & 0.022 & -0.136 & -0.092 \\
\hline Habit & & & & & & 0.106 & 0.020 & -0.196 & 0.135 \\
\hline Age & & & & & & & $-0.283^{*}$ & $0.422 * * *$ & $0.903^{* * * *}$ \\
\hline Gender & & & & & & & & $-0.436 * * *$ & $-0.278^{* *}$ \\
\hline Specialty & & & & & & & & & $.0403^{* * * *}$ \\
\hline
\end{tabular}

$* \mathrm{p}<0.05 ; * * \mathrm{p}<0.01 ; * * * \mathrm{p}<0.001$

teristics of participants. There are significant differences between the two groups of specialists. First, gender distribution is uneven, since women are generally a minority in orthopaedic surgery. Second, age distribution also is different between the two specialties, orthopaedics surgeons being older than ophthalmologists. Likewise, the mean clinical experience is higher among orthopaedic surgeons. These differences probably reflect a trend for specialty choice in younger cohorts of physicians where the proportion of women is higher [43].

Table 4 reports the descriptive statistics (means and standard deviations) of the theoretical variables. Normality of distribution and possible collinearity were assessed and results were satisfactory (see the research report for detailed results [32]). The mean value of the intention to use HTA recommendations is not markedly different between groups. However, all theoretical variables have a higher mean among ophthalmologists. The majority of theoretical variables have a mean value higher than 3 , which corresponds to a positive value. One exception is the variable habit that has a negative value (lower than 3 ) in both groups. Moreover, personal and social normative beliefs have a negative value among orthopaedic surgeons. These findings indicate that there might be significant differences between the two groups of specialists.

\section{Differences in intention to use HTA recommendations between specialties}

To assess the main effect difference between the two groups, i.e. how they globally differ on the set of theoretical variables, a multivariate analysis of variance (MANOVA) was conducted. This test allows for verifying equality of variances between multiple variables at the same time, without having to adjust for multiple testing. According to Hair et al.,[44] a MANOVA can be performed for uneven groups if the following three conditions are met: 1) the number of observations in the smallest group is higher than the number of dependent variables; 2 ) the number of observations in each group is higher than 20; and 3) there is a minimum of five observations for each dependent variable. All three conditions were met in this case.

The Hotelling's Trace was used to assess the main effect of medical specialty on the set of theoretical variables, as it has been recommended for two-groups MANOVA. [45] As shown in Table 4, the Hotelling's Trace test is significant, indicating that there is a global difference between groups. Furthermore, univariate tests show that all explicative variables of the model also are significantly different, except habit. However, the dependent variable of the model (intention) is not significantly different between groups.

Therefore, given that intention to use HTA recommendations to support decision-making might have had different determinants within each group of medical specialists, two logistical regression models were tested, including variables from the TIB and external variables.

\section{Factors influencing intention to use HTA recommendations for cataract surgery}

Table 5 presents the final regression model of the intention to use HTA recommendations for prioritisation of patients on waiting lists for cataract surgery. The model was significant and explained $87 \%$ of the variance (adjusted $\mathrm{R}^{2}$ ) in ophthalmologists' intention to use the HTA recommendations to support decision-making. The three determinants explaining this intention were, in order of importance: attitudinal beliefs $(\beta=0.40)$, personal normative beliefs $(\beta=0.36)$, and social normative beliefs $(\beta=0.25)$.

\section{Factors influencing intention to use HTA} recommendations for hip and knee replacement

The final regression model tested to explain the intention to use HTA recommendations for prioritisation of patients on waiting lists for hip and knee replacement is reported in Table 6. Again, the regression model was significant and explained $65 \%$ of the variance (adjusted $\mathrm{R}^{2}$ ) in orthopaedic surgeons' intention to use the recommen- 
Table 3: Sociodemographic and professional characteristics of respondents

\begin{tabular}{|c|c|c|c|}
\hline \multirow[t]{2}{*}{ Variable } & \multicolumn{2}{|c|}{ Medical specialty } & \multirow[t]{2}{*}{ Difference (chi-square or Student $t$-test) } \\
\hline & Ophthalmology & Orthopaedic surgery & \\
\hline \multicolumn{4}{|l|}{ Gender } \\
\hline Male (\%) & $19(54.3)$ & $51(83.6)$ & $\begin{array}{l}\chi^{2}=15.20 \\
p<0.001\end{array}$ \\
\hline Female (\%) & $9(25.7)$ & I (I.6) & \\
\hline Missing (\%) & $7(20.0)$ & $9(14.8)$ & \\
\hline \multicolumn{4}{|l|}{ Age group } \\
\hline$<30$ years $(\%)$ & $10(28.6)$ & $3(4.9)$ & $\begin{array}{l}\chi^{2}=19.95 \\
p<0.001\end{array}$ \\
\hline 30 - 39 years $(\%)$ & $12(34.3)$ & $9(14.8)$ & \\
\hline $40-49$ years $(\%)$ & $8(22.9)$ & $24(39.3)$ & \\
\hline$\geq 50$ years $(\%)$ & $5(14.3)$ & $25(41.0)$ & \\
\hline \multicolumn{4}{|l|}{ Clinical experience ${ }^{a}$} \\
\hline Mean & 11.0 & 18.7 & $t=4.22$ \\
\hline Standard deviation & \pm 8.4 & \pm 8.8 & $\mathrm{p}<0.001$ \\
\hline
\end{tabular}

a Two missing values

dations to support decision-making. The strongest predictors were facilitating conditions $(\beta=0.39)$, personal normative beliefs $(\beta=0.38)$, and habit $(\beta=0.25)$.

\section{Discussion}

This study was the first, to the best of our knowledge, to assess the psychological factors influencing physician intention to use HTA recommendations based upon a recognised theoretical framework. The TIB has been successful in explaining a variety of human behaviours, including the adoption of health technologies among healthcare professionals $[24,25,30,31]$. Using an established theoretical framework to assess the determinants of professional behaviours presents at least four advantages. First, it provides a basis for comparison between similar studies, thus supporting knowledge development in the field [46]. Second, it offers a sound methodological approach that improves the internal validity of studies based upon the advances in social psychology measurement. Third, it facilitates the realisation of systematic reviews in the field of implementation science [46]. Finally, it allows for the development of strategies to improve the success of inter- ventions to implement evidence-based practices [46-49]. This study also provides support to the cultural adaptability of a psychosocial theoretical framework such as the $\mathrm{TIB}$, since the items forming theoretical constructs were adapted to the specific context in which the study took place. This framework could thus be adapted and applied to a variety of settings in the field of implementation science.

A major finding of this study is that intention of physicians to use HTA recommendations in their practice is influenced by a different set of psychosocial factors, depending on the specific context. This difference can either be attributed to the characteristics of the technology targeted in the HTA recommendations, the social and cultural characteristics of the medical specialty, the specific context in which recommendations are implemented, or a combination of these factors. It would be necessary to study the adoption of various HTA recommendations across different medical specialties and contexts in order to verify these hypotheses.

Table 4: Main effect difference and differences in theoretical variables between medical specialties

\begin{tabular}{lllll}
\hline Theoretical variable & $\begin{array}{l}\text { Ophthalmology }(\mathrm{n}=35) \\
\text { Mean }(\mathrm{sd})\end{array}$ & $\begin{array}{l}\text { Orthopaedic surgery } \\
(\mathrm{n}=6 I) \text { Mean }(\mathrm{sd})\end{array}$ & $\begin{array}{l}\text { F-test for univariate } \\
\text { difference }(\mathrm{df})\end{array}$ \\
\hline Intention & $3.59( \pm I .13)$ & $3.49( \pm .87)$ & $\mathrm{F}=0.23(I, 94)$ & 0.63 \\
Attitudinal beliefs & $3.68( \pm .65)$ & $3.23( \pm .72)$ & $\mathrm{F}=9.50(I, 94)$ & 0.003 \\
Personal normative beliefs & $3.38( \pm I .14)$ & $2.91( \pm .77)$ & $\mathrm{F}=5.80(I, 94)$ & 0.018 \\
Social normative beliefs & $3.38( \pm .80)$ & $2.97( \pm .74)$ & $\mathrm{F}=6.43(I, 94)$ & 0.013 \\
Facilitating conditions & $4.26( \pm .75)$ & $3.59( \pm I .15)$ & $\mathrm{F}=9.45(I, 94)$ & 0.003 \\
Habit & $2.75( \pm I .24)$ & $2.3 I( \pm .96)$ & $\mathrm{F}=3.75(I, 94)$ & 0.056 \\
\hline
\end{tabular}

Hotelling's Trace $=0.336[F(6,89)=4.98 ; p<0.0001]$

* Considered significant at $\mathrm{p}<0.05$ 
Table 5: Regression of the intention to use HTA recommendations for prioritisation of patients on waiting lists for cataract surgery

\begin{tabular}{lcc}
\hline Theoretical variable & Standard estimate $(\beta)$ & $p$ value* \\
\hline Attitudinal beliefs & 0.40 & 0.001 \\
Personal normative beliefs & 0.36 & 0.004 \\
Social normative beliefs & 0.25 & 0.044 \\
\hline
\end{tabular}

$R^{2}$ of the model: $0.89[F(3,3 I)=77.44 ; p<0.00 I]$; Adjusted $R^{2}=0.87$

* Considered significant at $\mathrm{p}<0.05$

Nevertheless, the present study supports the need for mapping interventions to specific population groups in order to improve the adoption of evidence-based practices [50]. A previous study has reported limited impact of a tailored intervention aimed at introducing evidence-based practices among physicians, [51] but this lack of success was largely due to problems related to the implementation of the intervention [52].

Among the factors that were associated with intention to use HTA recommendations to support decision-making, personal normative beliefs were important in both groups of specialists. This variable was formed by three components, namely, personal norm, self-identity, and professional norm. The impact of personal morals or principles on clinical behaviours has been reported in a cross-cultural study of physicians' intention to prescribe hormone therapy [46]. The construct of professional norm, added to the TIB framework for this study, was found to influence physician intention to adopt telemedicine [24]. However, this is a relatively new concept that needs further psychometrical developments.

The influence of attitudinal beliefs on the intention to use HTA recommendations was significant only in the ophthalmologists group. Attitude has been found as an important determinant of clinical behaviours in other studies $[12,53]$. However, attitude was not associated with the intention of physicians to adopt telemedicine [24]. Thus, a positive perception of the benefits of using HTA recommendations was more important in explaining the intention to adopt the prioritisation system for cataract surgery. Borrowing a concept from the diffusion of innovation theory,[54] ophthalmologists who had the intention to use the prioritisation system were those who perceived a relative advantage to this innovation [55]. Hence, the decision to use the prioritisation system or not was mostly perceived as an individual choice. One plausible explanation is the fact that waiting lists were not perceived as a big issue for ophthalmologists, since most of them also performed cataract surgery in private practice. Thus, external pressure to adopt the prioritisation system was not as strong as for hip and knee replacement.

Facilitating conditions, i.e. factors in the environment that support the realisation of the behaviour, were the most influential determinant of intention to use HTA recommendations among orthopaedic surgeons. One plausible explanation is the fact that hip and knee replacements are more complex and costly procedures that require greater resources. Thus, the prioritisation system was endorsed by a majority of the departments as the 'local standard.' Furthermore, the variable habit was also associated with the intention to use HTA recommendations among orthopaedic surgeons, which supports the previous hypothesis. It is likely that individual healthcare professionals will tend to adopt evidence-based practice more easily when there is a supportive culture in the working environment. However, it is important to acknowledge a possible threat to professional autonomy when introducing explicit rationing policies, such as prioritisation systems for surgical procedures that can lead to resistance to change [56].

Table 6: Regression of the intention to use HTA recommendations for prioritisation of patients on waiting lists for hip and knee replacement

\begin{tabular}{lcc}
\hline Theoretical variable & Standard estimate $(\beta)$ & $p$ value* \\
\hline Facilitating conditions & 0.39 & 0.000 \\
Personal normative beliefs & 0.38 & 0.000 \\
Habit & 0.25 & 0.039
\end{tabular}

$\mathrm{R}^{2}$ of the model: $0.66[\mathrm{~F}(3,57)=37.40 ; \mathrm{p}<0.00 \mathrm{I}]$; Adjusted $\mathrm{R}^{2}=0.65$

* Considered significant at $\mathrm{p}<0.05$ 
Previous studies of the impact of HTA on decision-making at the health policy level recognize the difficulty of measuring how a specific recommendation would inform decision-making on a given topic $[57,58]$. Another contribution of this study is that it proposes a strategy to assess the impact of HTA recommendations at the clinical decision-making level. Of course, using behavioural intention as the dependent variable is a proxy for estimating actual behaviour, but the literature generally supports the concordance between intention and subsequent behaviour [59]. Recent efforts have been made to bridge the 'intention-behaviour gap.' For instance, moral factors, such as anticipated regret and moral norm have a significant impact on the consistence between intention and subsequent behaviour [60,61]. However, longitudinal studies are needed to assess the correspondence between physicians' intention to adopt evidence-based practices and their subsequent behaviours.

\section{Limitations of the study results}

Among the factors that may affect the possibility to generalise the results, it is important to mention a possible participation bias since respondents may have been more knowledgeable and/or interested in the HTA recommendations under study than non-respondents. Unfortunately, contacting non-respondents to assess this potential bias was not feasible since the study was anonymous.

The sample size was limited, despite a satisfactory response rate for this specific population. Previous studies usually report lower response rates for mail surveys among physicians $[62,63]$. The involvement of the Head of Department from each specialty in participating hospitals appeared as a successful strategy to improve participation in the study.

Given the small sample size, especially in the ophthalmologists group, it is important to use caution when interpreting the results. For undersized samples, the risk of unstable solution is greater when the independent variables are highly correlated. Also, a high $\mathrm{R}^{2}$ may reflect a problem of 'over-fitting,' i.e. a perfect but meaningless solution [64]. To test the stability of the solution in the ophthalmologists group, we verified if the pattern of the regression equation was affected by deleting the weakest predictor (social normative beliefs). The regression equation with the remaining two predictors (attitude and personal normative beliefs) was similar, indicating that the solution was stable. A multi-collinearity diagnosis was then performed. The variance inflation factors associated with independent variables were all below 10, showing no multicollinearity problem [44].
With respect to the possibility of over-fitting, other studies, both with small or larger samples, have reported high $\mathrm{R}^{2}$ in the prediction of behavioural intention among healthcare professionals based upon psychosocial theories $[24,46,65]$. In a study of physician intention to adopt telemedicine $(\mathrm{n}=506)$, a high $\mathrm{R}^{2}(.81)$ also was found, and similar correlations between the independent variables were present [24]. Thus we can conclude that the solution is likely to reflect a true relationship between the psychosocial predictors from the TIB and physician intention to use HTA recommendations.

A short vignette was used to bring physicians into a decision-making situation for which their intention to refer to the HTA recommendation was assessed. The vignette contained limited information on the clinical case and a hypothetical bias might have been present [66]. However, clinical vignettes are considered a valid and comprehensive method to asses the process of care provided in actual clinical practice [67]. Therefore, the findings of this study are likely to apply to 'real life' decision-making situations.

\section{Conclusion}

This study demonstrates the application of a social psychological model to understand the determinants of the adoption of evidence-based practices in healthcare. Of course, this represents only a small portion of the efforts needed to implement evidence-based interventions in order to improve quality in healthcare. Further work should address the translation of knowledge gained from studies on the determinants of healthcare professional behaviours into specific intervention strategies, the successful implementation of these strategies, and the evaluation of their effects on professional behaviours and, ultimately, on the effectiveness of the healthcare system.

\section{Competing interests}

The author(s) declare that they have no competing interests.

\section{Authors' contributions}

ES, JMVP and MPG participated in the design of the study. ES and MPG prepared the study questionnaires. MPG contacted the participants, proceeded to data collection and performed quantitative analyses. ES and JMVP reviewed the findings and a consensus was reached between all authors for data interpretation. MPG prepared a first draft of the manuscript and all authors revised and approved the last version of the manuscript.

\section{References}

I. Granados A: Health technology assessment and clinical decision making: which is the best evidence. Int J Technol Assess Health Care 1999, I 5(3):585-592.

2. Woolf $\mathrm{SH}$, Henshall $\mathrm{C}$ : Health technology assessment in the United Kingdom. Int J Technol Assess Health Care 2000, 16:591-625. 
3. A Scottish Health Technology Assessment Centre: Report of the Implementation Working Group. 1999 [http:// www.show.scot.nhs.uk/publications/me/imt/shtac.pdf].

4. Bero LA, Grilli R, Grimshaw JM, Harvey E, Oxman AD, Thomson MA Closing the gap between research and practice: an overview of systematic reviews of interventions to promote the implementation of research findings. $B M]$ 1998, 3 I 7:465-468.

5. Drummond $M$, Wheatherly $\mathrm{H}$ : Implementing the findings of health technology assessments. If the CAT got out of the bag, can the TAIL wag the dog. Int J Technol Assess Health Care 2000, 16(1): I-12.

6. Garcia-Altés A: La introduccion de tecnologias en los sistemas sanitarios: del dicho al hecho. Gaceta Sanitaria 2004, 1 8(5):398-405.

7. Granados A, Jonsson E, Banta HD, Bero L, Bonair A, Cochet C, Freemantle N, Grilli R, Grimshaw J, Harvey E, Levi R, Marshall D, Oxman A, Pasart L, Raisanen V, Ruis E, Espinas JA: EUR-ASSESS Project Subgroup Report on Dissemination and Impact. Int J Technol Assess Health Care 1997, 13(2):220-286.

8. Lehoux P, Denis JL, Tailliez S, Hivon M: Dissemination of Health Technology Assessments: Identifying the Visions Guiding an Evolving Policy Innovation in Canada. J Health Politics, Policy and Law 2005, 30(4):603-642.

9. Berg M, van der Grinten T, Klazinga N: Technology assessment, priority setting, and appropriate care in Dutch health care. Int J Technol Assess Health Care 2004, 20(I):35-43.

10. Hailey D: The influence of technology assessments by advisory bodies on health policy and practice. Health Policy 1993, 25(3):243-254.

II. Jacob R, McGregor M: Assessing the impact of health technology assessmen. Int J Technol Assess Health Care 1997, I3( I):68-80.

12. Sheldon TA, Cullum N, Dawson D, Lankshear A, Lowson K, Watt I, West $P$, Wright $D$, Wright J: What's the evidence that NICE guidance has been implemented? Results from a national evaluation using time series analysis, audit of patients' notes, and interviews. BMJ 2004, 329:999-1006.

13. Oliver A, Mossialos E, Robinson R: Health technology assessment and its influence on health care priority setting. Int J Technol Assess Health Care 2004, 20: I- 10.

14. Hivon M, Lehoux P, Denis J-L, Tailliez S: Use of health technology assessment in decision making: Coresponsibility of users and producers? Int J Technol Assess Health Care 2005, 2 I (2):266-275.

15. McGregor M, Brophy JM: End-user involvement in health technology assessment (HTA) development: a way to increase impact. Int J Technol Assess Health Care 2005, 21 (2):263-267.

16. Juzwishin D, Olmstead D, Menon D: Hospital-based technology assessment programmes: two Canadian examples. World Hosp Health Serv 1996, 32(2):2-9.

17. Lee RC, Marshall D, Waddell C, Hailey D, Juzwishin D: Health technology assessment, research, and implementation within a health region in Alberta, Canada. Int J Technol Assess Health Care 2003, 19(3):513-20.

18. Agence d'Evaluation des Technologies et des Modes d'Intervention en Santé (AETMIS): Health technology assessment in teaching hospitals. Government of Quebec 2003 [http://www.aet
a mis.gouv.qc.ca/site/down

load.php?52fl4aeb2244c6af235a383d92ec7bf4]

19. Grimshaw JM, Thomas RE, MacLennan G, Fraser C, Ramsay CR, Vale L, Whitty P, Eccles MP, Matowe L, Shirran L, Wensing M, Dijkstra R, Donaldson C: Effectiveness and efficiency of guideline dissemination and implementation strategies. Health Technol Assess 2004, 8(30):iii-iv. I-207

20. Triandis HC: Values, attitudes and interpersonal behaviour. In Nebraska Symposium on Motivation, 1979: Beliefs, attitudes and values Edited by: Page MM. Lincoln, University of Nebraska Press; 1980.

21. Ajzen I: The theory of planned behaviour. Organ Behav Hum Dec I991, 50:179-2II.

22. Bandura A: Self-efficacy: Toward a unifying theory of behavioral change. Psychol Rev 1977, 84(2): $191-215$.

23. Facione NC: The Triandis model for the study of health and illness behavior: A social behavior theory with sensitivity to diversity. Adv Nurs Sci 1993, 15:49-58.

24. Gagnon M-P, Godin G, Gagné C, Fortin J-P, Lamothe L, Reinharz D, Cloutier A: An adaptation of the Theory of Interpersonal Behaviour to the study of telemedicine adoption by physicians. Int J Med Inform 2003, 7I(2-3): I03-II5.
25. Bergeron F, Raymond L, Rivard S, Gara M: Determinants of EIS use: Testing a behavioral model. Dec Support Syst 1995, 14:| $13 \mid-146$

26. Honkanen P, Olsen SO, Verplanken B: Intention to consume seafood. Appetite 2005, 45:161-168.

27. Ouellette JA, Wood W: Habit and intention in everyday life: The multiple processes by which past behaviour predicts future behaviour. Psychol Bull 1998, I 24(I):54-74.

28. Jorm C, Kam P: Does medical culture limit doctors' adoption of quality improvement? Lessons from Camelot. J Health Serv Res 2004, 9(4):248-25I.

29. Triandis HC: The self and social behavior in differing cultural contexts. Psychol Rev 1989, 96:506-520.

30. Paré G, Elam J: Discretionary use of personal computers by knowledge workers: Testing of a social psychology theoretical model. Behavior \& Information Technology 1995, 14:215-218.

31. Thompson RL, Higgins CA, Howell JM: Personal computing: Towards a conceptual model of utilization. MIS Quarterly I99I, 15:125-142.

32. Gagnon MP, Sanchez E, Pons JMV: El impacto de las recomendaciones basadas en la evaluación de tecnologías médicas sobre la práctica clínica y organizacional. Agència d'Avaluatió de Tecnologia y Recerca Mèdiques, Barcelona 2005 [http://www.gencat.net/ salut/depsan/units/aatrm/pdf/in050les.pdf]

33. Gagnon MP, Sanchez E, Pons JMV: The integration of Health Technology Assessment (HTA) recommendations into organisational and clinical practice: A case study in Catalonia. Int J Technol Assess Health Care 22(2):. forthcoming

34. Kimberly JR, Evanisko MJ: Organizational innovation: The influence of individual, organizational and contextual factors on hospital adoption of technological and administrative innovations. Acad Manage J 198I, 24(4):689-7I3.

35. Fishbein M, Bandura A, Triandis HC, Kanfer FH, Becker MH, Middlestadt SE, Eichler A: Factors influencing behavior and behavior change: Final report-theorist's workshop Rockville (MD), National Institute of Mental Health; 1992.

36. Gagné C, Godin G: The theory of planned behavior: Some measurement issues concerning belief-based variables. J Appl Soc Psychol 2000, 30:2173-2193.

37. Pelto PJ: Anthropological Research: The structure of inquiry New York, Harper \& Row; 1970

38. Davidson AR, Jaccard J], Triandis HC, Morales ML, Diaz-Guerrero R: Cross-cultural model testing toward a solution of the eticemic dilemma. Intern J Psychol 1976, I I:I-I3.

39. Hobbis ICA, Sutton S: Are Techniques Used in Cognitive Behaviour Therapy Applicable to Behaviour Change Interventions Based on the Theory of Planned Behaviour? J Health Psychol 2005, 10:7-I8.

40. Nunnally JM: Psychometric Theory New York, McGraw Hill; 1978.

4l. Pedhazur EL: Multiple regression in behavioral research: Explanation and Prediction 2nd edition. New York, Holt, Rinehart \& Winston; 1982.

42. Hosmer DW, Lemeshow S: Applied logistic regression New York, Wiley \& Sons; 1989.

43. Buddeberg-Fischer B, Klaghofer R, Abel T, Buddeberg K: The influence of gender and personality traits on the career planning of Swiss medical students. Swiss Med Wkly 2003, 133:535-540.

44. Hair JF, Anderson RE, Tathum RL, Black WC: Multivariate Data Analysis with Readings New York, Macmillan; 1998.

45. Lowery SE, Robinson Kurpius SE, Befort C, Hull Blanks E, Foley Nicpon M, Sollenberger S, Huser L: Body Image, Self-Esteem, and Health-Related Behaviors Among Male and Female First Year College Students. J Coll Student Dev 2005, 46(6):6|2-623.

46. Légaré F, Godin G, Ringa V, Dodin S, Turcot L, Norton J: Variation in the psychosocial determinants of the intention to prescribe hormone therapy prior to the release of the Women's Health Initiative trial: a survey of general practitioners and gynaecologists in France and Quebec. BMC Med Inform Dec Making 2005, 5:31.

47. Eccles M, Grimshaw J, Walker A, Johnston M, Pitts N: Changing the behavior of healthcare professionals: the use of theory in promoting the uptake of research findings. I Clin Epidemiol 2005, 58(2): $107-1 \mid 2$.

48. Michie S, Johnston M, Abraham C, Lawton R, Parker D, Walker A: Making psychological theory useful for implementing evidence based practice: a consensus approach. Qual Saf Health Care 2005, I4(I):26-33. 
49. Walker AE, Grimshaw J, Johnston M, Pitts N, Steen N, Eccles M: PRIME - PRocess modelling in ImpleMEntation research: selecting a theoretical basis for interventions to change clinical practice. BMC Health Serv Res 2003, 3(I):22.

50. Bartolomew LK, Parcel GS, Kok G: Intervention mapping: A process for developing theory- and evidence-based health education programs. Health Educ Behav 1998, 25(5):545-563.

5I. Flottorp S, Oxman AD, Havelsrud K, Treweek S, Herrin J: Cluster randomised controlled trial of tailored interventions to improve the management of urinary tract infections in women and sore throat. BMJ 2002, 325(7360):367.

52. Flottorp S, Havelsrud K, Oxman AD: Process evaluation of a cluster randomized trial of tailored interventions to implement guidelines in primary care - why is it so hard to change practice? Fam Pract 2003, 20(3):333-339.

53. Liabsuetrakul T, Chongsuvivatwong $\mathrm{V}$, Lumbiganon P, Lindmark G: Obstetricians' attitudes, subjective norms, perceived controls, and intentions on antibiotic prophylaxis in caesarean section. Soc Sci Med 2003, 57(9): 1665-1674.

54. Rogers EM: Diffusion of Innovations Fifth edition. New York, The Free Press; 2003.

55. Berwick DM: Disseminating innovations in health care. JAMA 2003, 289: 1969-1975

56. Mechanic D: Muddling through elegantly: Finding the proper balance in rationing. Health Affair 1997, 5:83-92.

57. Dixon S, Coleman P, Nicholl J, Brennan A, Touch S: Evaluation of the impact of a technology appraisal process in England: the South and West Development and Evaluation Committee. J Health Serv Res Policy 2003, 8(I): 18-24.

58. Leys M: Healthcare policy: Qualitative evidence and health technology assessment. Health Policy 2003, 65:217-26.

59. Armitage C], Conner M: Efficacy of the Theory of Planned Behaviour: A meta-analytic review. Brit J Soc Psychol 200I, 40(4):47I-499.

60. Abraham C, Sheeran P: Acting on intentions: The role of anticipated regret. Brit J Soc Psychol 2003, 42:495-5II.

61. Godin G, Connor M, Sheeran P: Bridging the intention-behaviour "gap": The role of moral norm. Brit J Soc Psychol 2005, 44:497-5 I2.

62. Leung GM, Ho LM, Chan MF, Johnston MJ, Wong FK: The effects of cash and lottery incentives on mailed surveys to physicians: a randomized trial. J Clin Epidemiol 2002, 55:80I-807.

63. Kellerman SE, Herold J: Physician response to surveys: A review of the literature. Am J Prev Med 200I, 20(1):6I-67S

64. Tabachnik BG, Fidell LS: Using multivariate statistics Third edition. New York, Harper Collins College Publishers; 1996.

65. Daneault S, Beaudry M, Godin G: Psychosocial determinants of the intention of nurses and dietitians to recommend breastfeeding. Can J Public Health 2004, 95(2): I5I-I54.

66. Aizen I, Brown TC, Carvajal F: Explaining the Discrepancy between Intentions and Actions: The Case of Hypothetical Bias in Contingent Valuation. Pers Soc Psychol B 2004, 30(9): $|108-||2|$.

67. Peabody JW, Luck J, Glassman P, Dresselhaus TR, Lee M: Comparison of vignettes, standardized patients, and chart abstraction: a prospective validation study of 3 methods for measuring quality. JAMA 2000, 283: $1715-1722$.
Publish with Biomed Central and every scientist can read your work free of charge

"BioMed Central will be the most significant development for disseminating the results of biomedical research in our lifetime. "

Sir Paul Nurse, Cancer Research UK

Your research papers will be:

- available free of charge to the entire biomedical community

- peer reviewed and published immediately upon acceptance

- cited in PubMed and archived on PubMed Central

- yours - you keep the copyright
BioMedcentral 